\title{
Reconstitution de dose par calcul Monte Carlo en cas d'accident radiologique : application à l'accident du Chili de décembre 2005
}

\author{
C. HUET $^{1}$, I. CLAIRAND ${ }^{1}$, F. TROMPIER ${ }^{1}$, E. BEY ${ }^{2}$, J.-F. BOTTOLLIER-DEPOIS ${ }^{1}$
}

(Manuscrit reçu le 5 mars 2007, accepté le 16 juillet 2007)

RÉSUMÉ À la suite d'un accident radiologique dû à une source de gammagraphie survenu au Chili en décembre 2005 et impliquant une victime, l'IRSN a été sollicité pour réaliser la reconstitution dosimétrique par calcul Monte Carlo. Des outils, développés au laboratoire, associant des fantômes anthropomorphes mathématiques et voxélisés au code de calcul Monte Carlo monpx ont été mis en ouvre afin de déterminer la distribution de dose au niveau de la fesse gauche de la victime et la dose aux organes critiques. La cartographie dosimétrique réalisée au niveau de la fesse gauche montre que la dose à la surface est importante $(1900 \mathrm{~Gy})$ et chute rapidement pour atteindre $20 \mathrm{~Gy}$ à $5 \mathrm{~cm}$ de profondeur. Les calculs réalisés avec un fantôme mathématique indiquent que les doses moyennes aux organes critiques sont faibles et permettent d'identifier de possibles sites de prélèvement de moelle osseuse. S'appuyant sur la cartographie dosimétrique, une exérèse de $5 \mathrm{~cm}$ de profondeur et $\mathrm{de} 10 \mathrm{~cm}$ de diamètre a été pratiquée au niveau de la fesse gauche de la victime.

ABSTRACT Monte Carlo dose reconstruction in case of a radiological accident: application to the accident in Chile in December 2005.

Following a radiological accident caused by a gammagraphy source in Chile in December 2005 involving one victim, IRSN was contacted to perform the dosimetric reconstruction of the accident using numerical simulation. Tools developed in the laboratory, associating anthropomorphic mathematic or voxel phantoms with the Monte Carlo calculation code monpx, were used in order to determine the dose distribution on the left buttock and absorbed doses to critical organs. The dosimetric mapping show that the absorbed at the skin surface is very high $(1900 \mathrm{~Gy})$ but drops rapidly at deep. At a depth of $5 \mathrm{~cm}$, it is $20 \mathrm{~Gy}$. Calculations performed with a mathematical phantom indicate that average doses to the critical organs are relatively low. Moreover, possible bone marrow sites for puncture are identified. Based on the dosimetric mapping, an excision measuring $5 \mathrm{~cm}$ in depth by $10 \mathrm{~cm}$ in diameter was performed on the left buttock of the victim.

Keywords: radiological accident / dose reconstruction / Monte Carlo calculation

1 IRSN, Direction de la radioprotection de l'Homme, Service de dosimétrie externe, B.P. 17, 92262 Fontenay-aux-Roses Cedex, France.

2 Hôpital d'Instruction des Armées Percy, B.P. 410, 92141 Clamart Cedex, France. 


\section{Introduction}

La diversité et la complexité des accidents radiologiques rendent difficiles le choix de la thérapeutique et le pronostic médical à partir des seules observations cliniques. Le Laboratoire de dosimétrie des rayonnements ionisants (LDRI) de l'IRSN développe des outils permettant la reconstitution d'accidents radiologiques dus à une source externe par simulation numérique. Ces outils ont été appliqués, en temps réel, à un accident survenu au Chili en décembre 2005.

Le 15 décembre 2005, au Chili, un ouvrier a ramassé une source d ${ }^{192}$ Ir tombée par inadvertance d'un appareil de gammagraphie. Il l'a manipulée à mains nues et également placée dans la poche arrière gauche de son pantalon avant qu'elle ne soit détectée par une personne munie d'un dosimètre électronique environ 40 minutes après sa découverte par l'ouvrier. Très rapidement, un érythème est apparu sur la fesse gauche de la victime. Le Chili a demandé l'intervention de l'AIEA qui a mandaté plusieurs personnes sur place. La victime a été transportée en France le 29 décembre 2005 pour être soignée à l'hôpital d'Instruction des Armées Percy de Clamart. Le LDRI a été sollicité dès le 19 décembre pour la reconstitution dosimétrique de l'accident par simulations numériques; cet article décrit les techniques de simulation numérique utilisées pour la reconstitution de dose de cet accident et présente les résultats.

\section{Données utilisées}

Les données utilisées pour effectuer la reconstitution dosimétrique ont été tirées de l'enquête réalisée par les autorités chiliennes compétentes ainsi que du rapport fait par les personnes mandatées sur place par l'AIEA.

La source est une source d ${ }^{192}$ Ir cylindrique de $1 \mathrm{~mm}$ de rayon sur $2 \mathrm{~mm}$ de hauteur encapsulée dans un cylindre en acier de $2 \mathrm{~mm}$ d'épaisseur sur $6 \mathrm{~mm}$ de hauteur. Une photo de la source est présentée sur la figure 1. L'activité de la source au moment de l'accident était de 3,3 TBq $(90 \mathrm{Ci}) . \mathrm{L}^{, 192} \mathrm{Ir}$ est un métal très dense, sa période radioactive est de 74 jours. Il émet par désintégration des particules bêta moins d'énergie 256, 590 et $670 \mathrm{keV}$ mais surtout un rayonnement complexe de plusieurs raies gamma d'énergie moyenne $350 \mathrm{keV}$.

D'après le scénario s'appuyant principalement sur le témoignage de la victime, cette dernière a manipulé la source pendant plusieurs minutes à mains nues en la faisant passer d'une main à l'autre. La source a également été placée pendant une dizaine de minutes dans la poche arrière gauche de son pantalon. Enfin, elle est restée dans la poche gauche de sa veste pendant un temps indéterminé. 


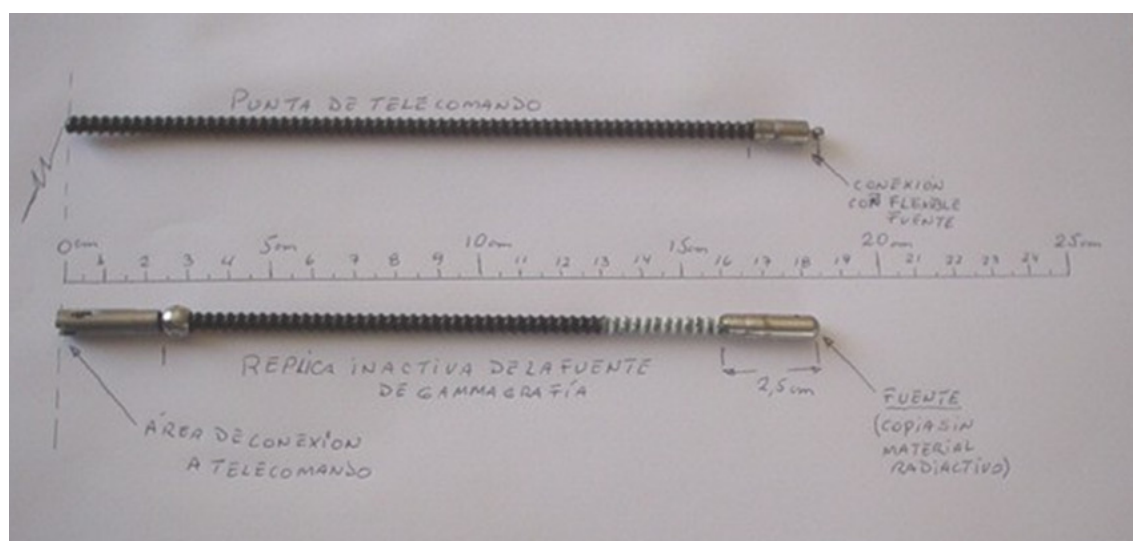

Figure 1 - Photographie de la source ${ }^{, 192}$ Ir à l'origine de l'accident (source de gammagraphie). Photograph of the ${ }^{192}$ Ir source that caused the accident (gammagraphy source).

\section{Matériels et méthodes}

Dans cette étude, plusieurs configurations ont été modélisées afin de décrire au mieux les différentes séquences du scénario. Cependant, l'exposition au niveau des mains n'a pas pu être évaluée car le scénario (position de la source, temps) était trop imprécis. Les calculs ont été réalisés avec le code Monte Carlo d'interaction rayonnement - matière MCNPX version 2.4.0 (Waters, 2002). Dans toutes les configurations, seule la composante photonique de la source d ${ }^{192} \operatorname{Ir}$ a été prise en compte étant donnée la géométrie de la source.

\subsection{Source placée dans la poche arrière gauche du pantalon}

\subsubsection{Calculs avec une géométrie simplifiée}

Dans un premier temps, il s'agissait de fournir rapidement une estimation de la dose en profondeur au niveau de la fesse gauche. La fesse a été modélisée par une sphère de tissu mou de $25 \mathrm{~cm}$ de diamètre et les calculs de dose en profondeur et en surface ont été réalisés en considérant la source à $2 \mathrm{~mm}$ de la surface de la peau et un temps d'exposition de 10 minutes. Nous avons également réalisé des calculs avec des temps d'exposition de 5, 15 et 20 minutes afin d'étudier la variation des isodoses. En effet, étant donné que le temps d'exposition (10 minutes) est basé sur le témoignage de la victime, nous avons voulu étudier l'influence, sur la dose en profondeur, de l'incertitude liée au temps d'exposition. 


\subsubsection{Calculs avec un fantôme anthropomorphe mathématique}

Des calculs ont été réalisés en parallèle à l'aide du fantôme numérique utilisé au laboratoire. Il s'agit du fantôme anthropomorphe mathématique développé par Cristy et Eckerman (1987) auquel quelques modifications ont été apportées (Furstoss et Ménard, 2005) (Fig. 2). Le but de ces calculs était de déterminer la dose en quelques points susceptibles d'être des sites de prélèvement de moelle osseuse, ainsi que la dose moyenne à certains organes critiques situés à proximité de la source. Nous avons également considéré un temps d'exposition de 10 minutes.
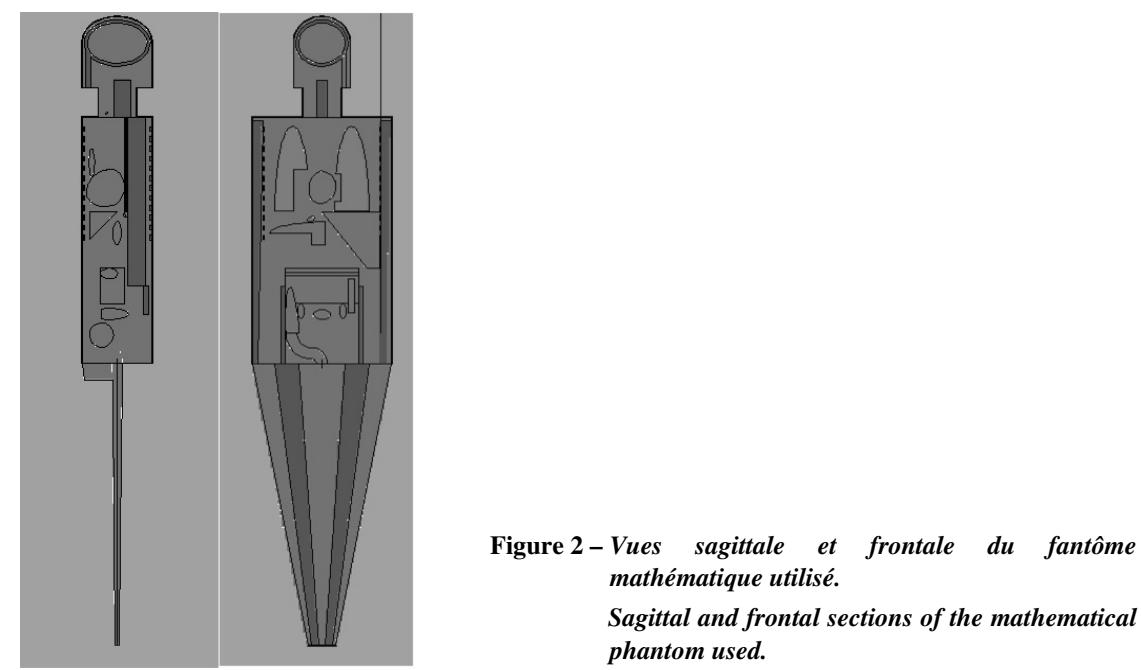

\subsubsection{Calculs avec un fantôme anthropomorphe voxélisé}

Suite à l'hospitalisation de la victime en France, nous avons pu obtenir les images du scanner et de l'IRM réalisés à l'hôpital Percy. Nous avons sélectionné 163 coupes allant du milieu de l'abdomen jusqu'à mi-cuisses. Ensuite, à l'aide du logiciel SESAME (Lemosquet et al., 2004), nous avons généré un fantôme voxélisé comportant les contours externes et les structures osseuses, et défini et positionné la source (Fig. 3). Enfin, nous avons sélectionné la zone sur laquelle nous souhaitions réaliser un calcul d'isodoses, c'est-à-dire au niveau du centre de la lésion. Une représentation 3D du fantôme voxélisé et de la source est illustrée sur la figure 4. 


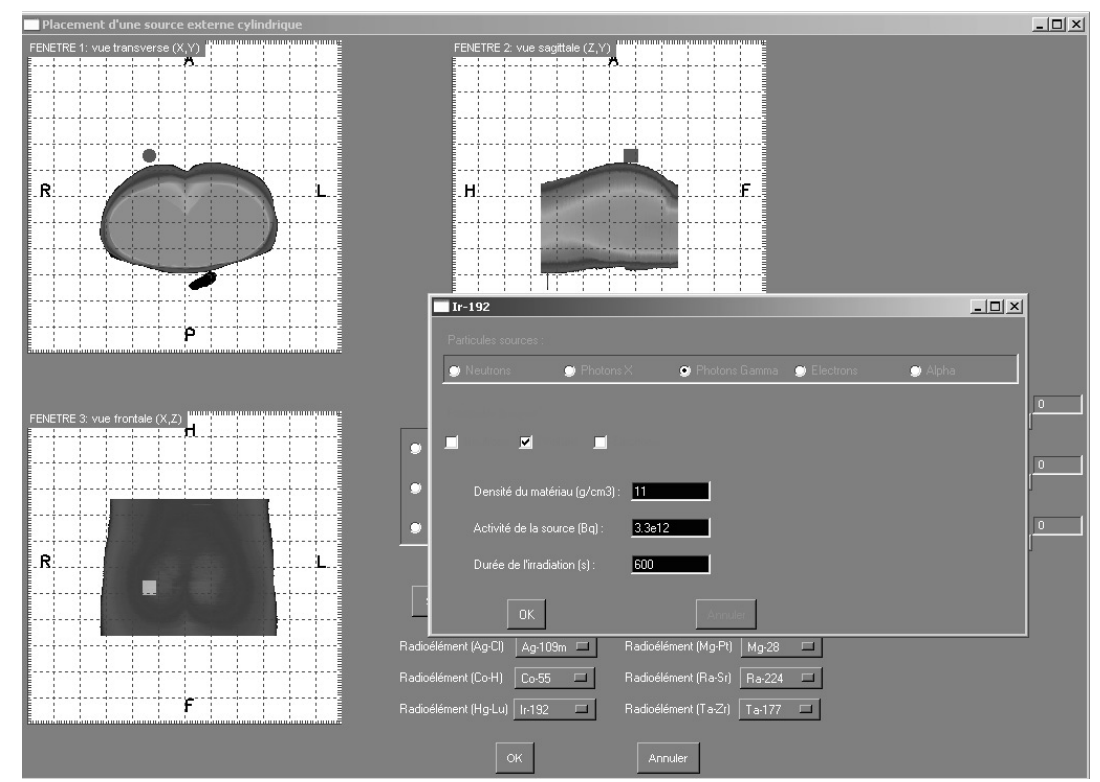

Figure 3 - Positionnement et définition de la source avec SESAME. Position and definition of the source using SESAME.

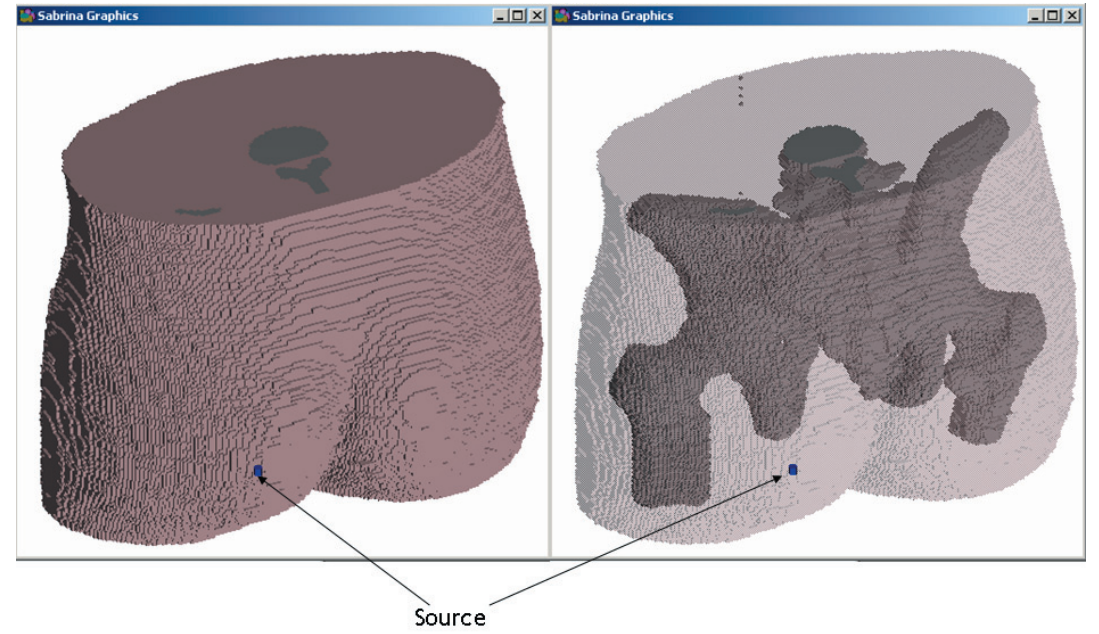

Figure 4 - Visualisation 3D du fantôme voxélisé (enveloppe externe et squelette) et de la source à l'aide du logiciel SABRINA (Van Riper, 1997).

$3 D$ View of the voxel phantom (external envelope and skeleton) and of the source using SABRINA software (Van Riper, 1997). 


\subsection{Source placée dans la poche de la veste}

Dans la configuration de la source placée dans la poche de la veste, les calculs ont également été réalisés avec la version modifiée du fantôme de Cristy et Eckerman. La source a été placée au niveau de la poitrine à $2 \mathrm{~cm}$ de la surface de la peau. Nous avons calculé :

- la dose moyenne pour différents organes,

- la dose en quelques points susceptibles d'être des sites de prélèvement de moelle osseuse.

Dans cette configuration, nous ne disposons pas du temps d'exposition. Étant donné qu'aucun signe clinique n'est apparent sur la poitrine, nous avons considéré que la dose peau est inférieure ou égale à 5 Gy. Nous avons donc déduit le temps d'exposition à partir du débit de dose calculé à la surface de la peau en considérant que la dose était de 5 Gy.

\section{Résultats}

\subsection{Source placée dans la poche arrière gauche du pantalon}

\subsubsection{Calculs avec une géométrie simplifiée}

\subsubsection{Calcul avec un temps d'exposition de 10 minutes}

Les résultats obtenus dans cette configuration sont présentés dans le tableau I. À la surface de la peau, la dose absorbée est très importante (1900 Gy) mais elle chute rapidement en profondeur à cause de l'effet combiné de la distance et de l'atténuation

\section{TABLEAU I}

Doses en profondeur et à la surface de la peau. Deep doses and doses at the skin surface.

\begin{tabular}{|c|c|c|c|}
\hline \multicolumn{2}{|c|}{ Dose en profondeur } & \multicolumn{2}{|c|}{ Dose à la surface de la peau } \\
\hline cm & Gy & cm & Gy \\
\hline 0,1 & 1900 & 0 & 2000 \\
\hline 1 & 520 & 0,3 & 1300 \\
\hline 3,3 & 50 & 0,75 & 680 \\
\hline 4,5 & 25 & 1,35 & 300 \\
\hline 7,2 & 10 & 2 & 170 \\
\hline 10 & 5 & 3 & 60 \\
\hline 14,5 & 2 & 5,5 & 17 \\
\hline 19 & 1 & 8 & 5 \\
\hline
\end{tabular}




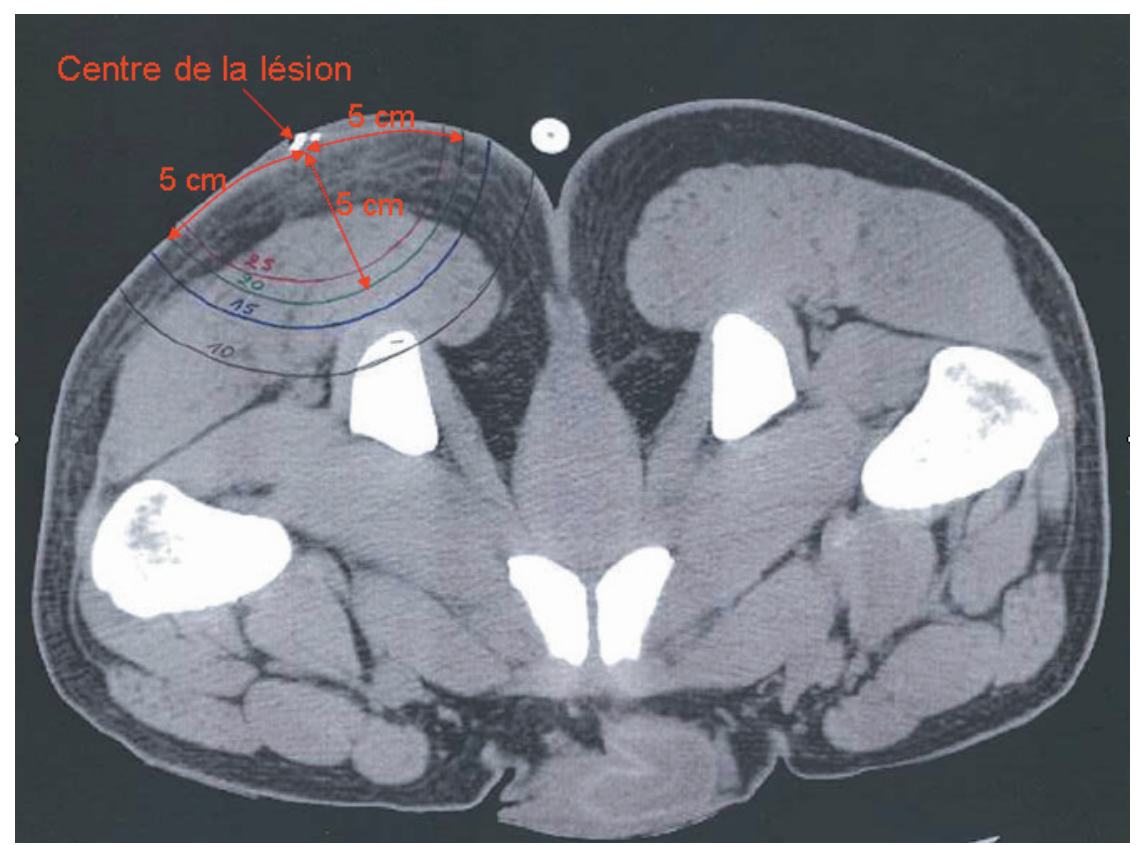

Figure 5 - Superposition des isodoses (valeurs en Gy) sur la coupe scanner de la victime. Isodoses (values in Gy) superimposed over the victim's CT slice.

dans les tissus. À $5 \mathrm{~cm}$, elle est de 20 Gy. La figure 5 représente les isodoses obtenues superposées à l'image scanner obtenue au niveau du plan de la source.

\subsubsection{Calculs avec d'autres temps d'exposition}

Nous nous sommes plus particulièrement intéressés à la position de l'isodose $20 \mathrm{~Gy}$ car elle constitue la valeur limite de nécrose des tissus. La variation en profondeur de l'isodose $20 \mathrm{~Gy}$ pour les différents temps d'exposition est présentée sur la figure 6 . L'isodose 20 Gy est située à $3,7 \mathrm{~cm}$ pour 5 minutes d'exposition et à $7 \mathrm{~cm}$ pour 20 minutes d'exposition. En outre, les valeurs de la dose à $5 \mathrm{~cm}$ de profondeur (position de l'isodose $20 \mathrm{~Gy}$ pour un temps d'exposition de 10 minutes) pour les différents temps d'exposition ont été calculées. On observe une variation entre 10 Gy pour 5 minutes d'exposition et 40 Gy pour 20 minutes d'exposition.

\subsubsection{Calculs avec un fantôme mathématique}

Les doses absorbées moyennes à certains organes dans cette configuration de calcul sont données dans le tableau II. Elles sont relativement faibles du fait que la source était assez éloignée de ces organes d'après le scénario retenu. 


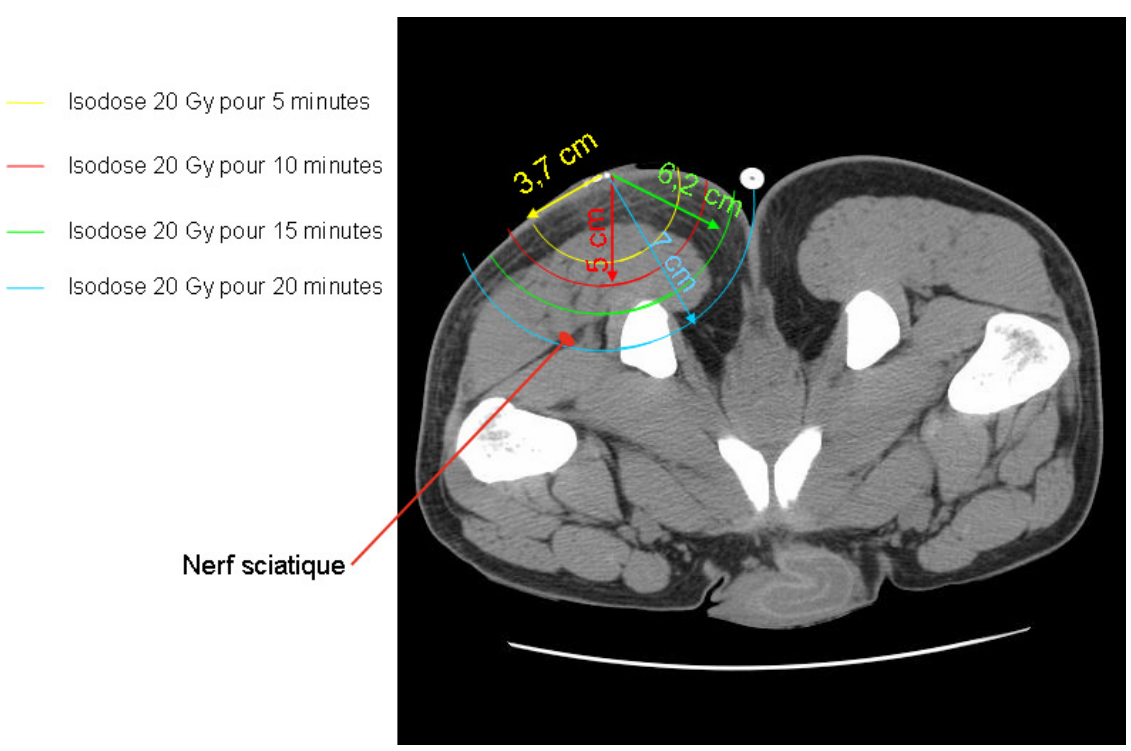

Figure 6 - Position de l'isodose 20 Gy pour différents temps d'exposition.

Position of the 20 Gy isodose for different exposure times.

Tableau II

Doses moyennes aux différents organes obtenues avec le fantôme mathématique en considérant la source dans la poche arrière gauche du pantalon au niveau de la lésion pendant $\mathbf{1 0}$ minutes.

Average doses to different organs obtained using the mathematical phantom with the source in the back left-hand trouser pocket on a level with the lesion for a period of $\mathbf{1 0}$ minutes.

\begin{tabular}{cc}
\hline Organe & Dose moyenne pour $\mathbf{1 0}$ minutes d'exposition $(\mathbf{G y})$ \\
\hline Reins & 0,3 \\
Vessie & 1,1 \\
Colon & 1,2 \\
Estomac & 0,2 \\
\hline
\end{tabular}

Les résultats du calcul de dose en différents points du haut du pelvis à une hauteur de $10 \mathrm{~cm}$ au-dessus du plan de la source sont présentés sur la figure 7 . On observe des valeurs allant de 4,1 Gy pour le point le plus proche de la source à 0,3 Gy pour le point le plus éloigné. Ces indications ont été utilisées pour effectuer le prélèvement de moelle osseuse. 


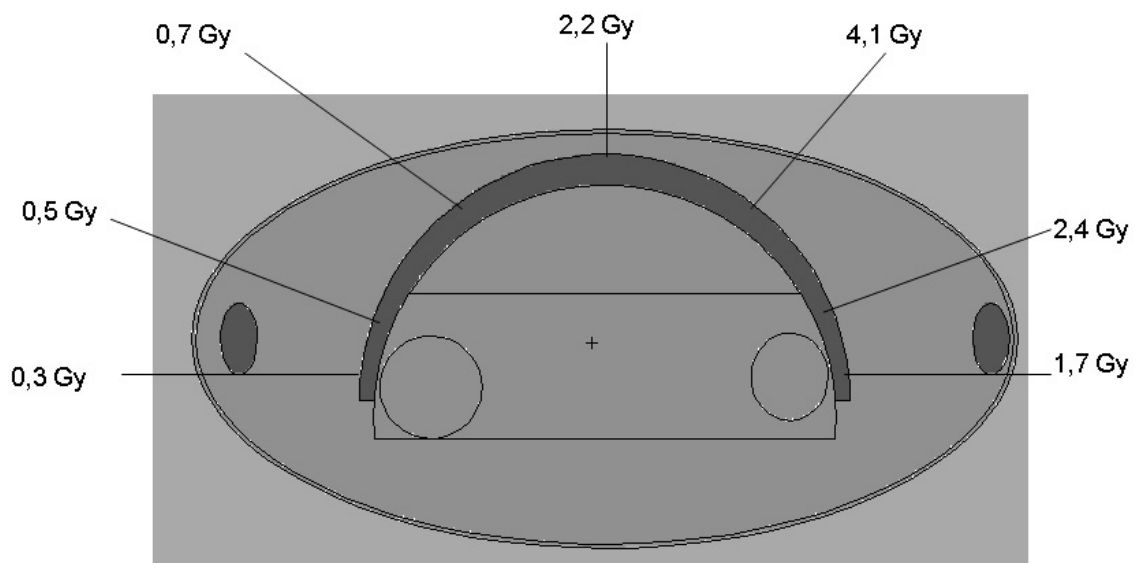

Figure 7 - Doses moyennes en quelques points susceptibles d'être des sites de prélèvement de moelle osseuse.

Average doses at certain points likely to be good bone marrow sampling sites.

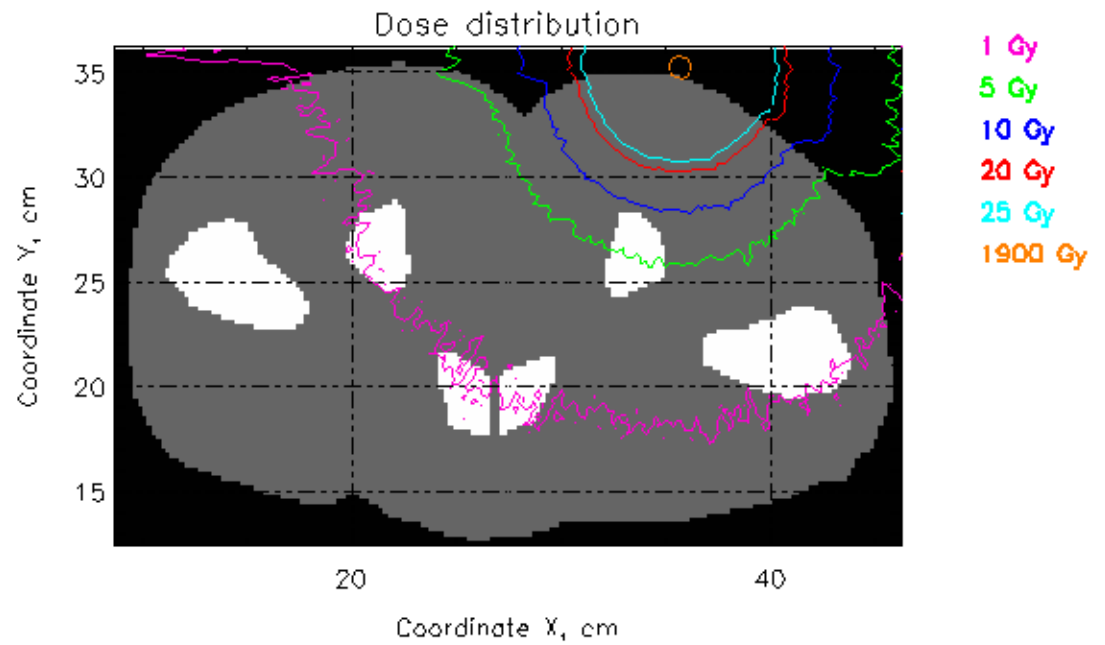

Figure 8 - Distribution de dose obtenue au niveau du plan de la source avec le fantôme voxélisé.

Dose distribution obtained on the same level as the source plane using the voxel phantom.

\subsubsection{Calculs avec un fantôme voxélisé}

Les résultats des isodoses obtenues avec le fantôme voxélisé sont comparables à ceux obtenus avec le modèle simplifié de la sphère (Fig. 8). Jusqu'à l'isodose 5 Gy, 
on observe une totale symétrie entre la dose en profondeur et la dose en surface : les isodoses sont en effet décrites par des arcs de cercle. Nous observons cependant un léger décalage des isodoses entre le modèle simplifié et le fantôme voxélisé, dû aux différences géométriques entre les deux configurations (os non représenté dans le modèle simplifié, courbure de la fesse légèrement différente, etc.). Ce décalage est de $6 \mathrm{~mm}$ pour l'isodose 10 Gy et d'à peine $2 \mathrm{~mm}$ pour l'isodose $20 \mathrm{~Gy}$. Ces écarts sont tout à fait acceptables étant données les incertitudes liées au temps d'exposition et permettent de valider le modèle simplifié dans ce cas.

\subsection{Source placée dans la poche de la veste}

Dans cette configuration de calcul, le débit de dose à la peau est de $500 \mathrm{~Gy} \mathrm{~h}^{-1}$. Pour que la dose à la peau n'excède pas 5 Gy, dose à partir de laquelle des signes cliniques devraient apparaître, le temps maximal d'exposition a été estimé à 40 secondes. Dans la suite des résultats, nous avons donc considéré que la source est restée 40 secondes dans la poche de la veste de la victime.

Les doses moyennes aux organes proches de la source (poumons et cœur) sont faibles. Les résultats obtenus pour les sites de prélèvement de moelle osseuse sont présentés sur la figure 9. Les doses absorbées, pour un temps d'exposition de 40 secondes, sont faibles.

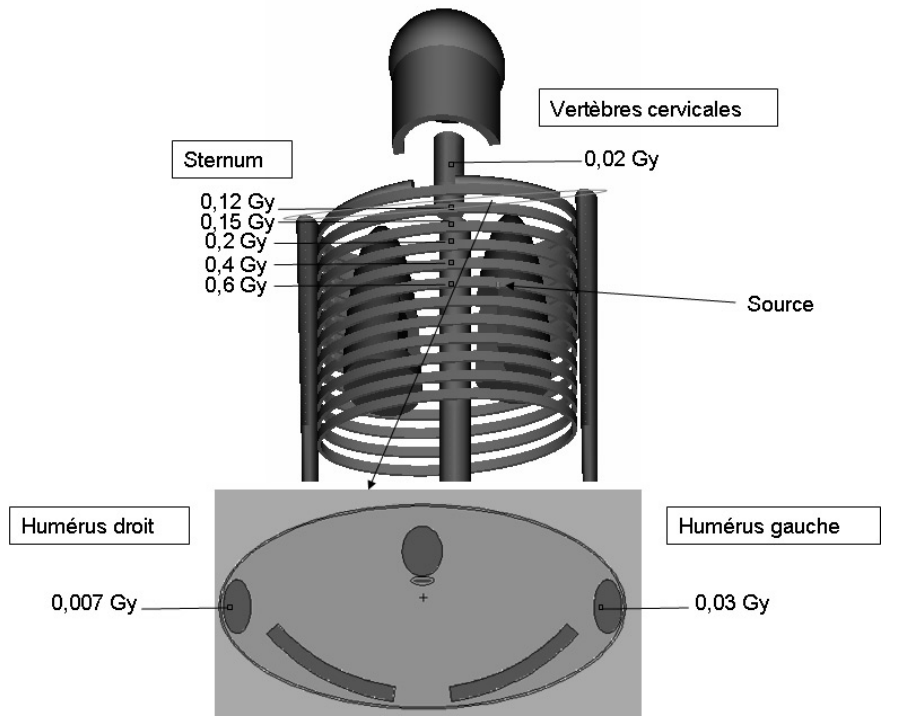

Figure 9 - Doses sur les sites de moelle osseuse.

Doses to bone marrow sites. 


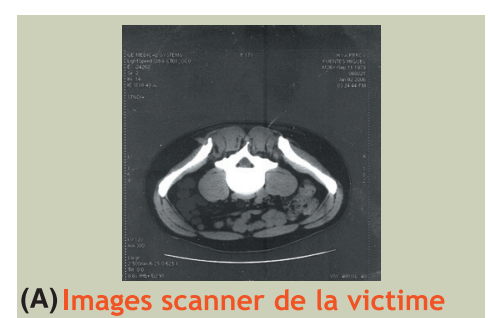

(A) Images scanner de la victime

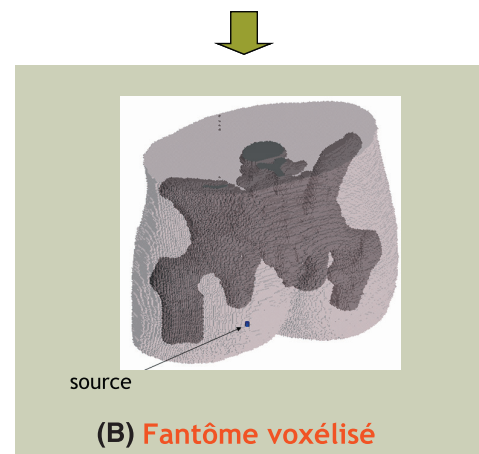

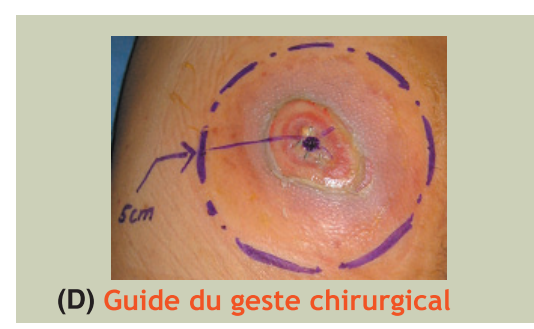

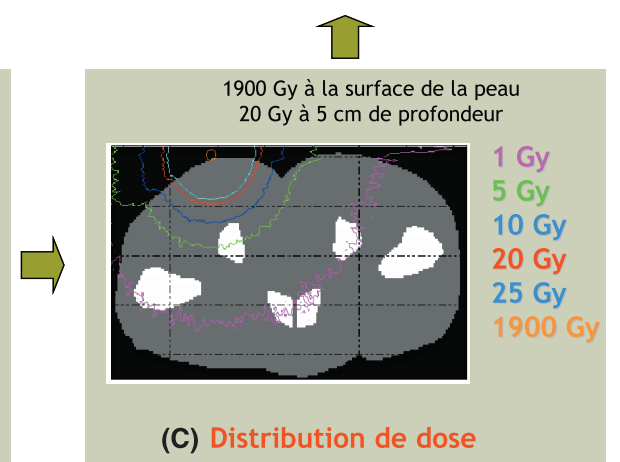

Figure 10 - Reconstitution dosimétrique avec SESAME : guide du geste chirurgical. (A) Images scanner de la victime : 163 coupes allant du milieu de l'abdomen jusqu'à mi-cuisses ont été utilisées pour générer un fantôme numérique voxélisé personnalisé. (B) Vue du fantôme voxélisé et de la source. Grâce au logiciel SESAME, un fantôme voxélisé comportant les contours externes et les structures osseuses a été généré et la source $d^{192}$ Ir a été positionnée à $2 \mathrm{~mm}$ du centre de la lésion située sur la fesse gauche. (C) Distribution de dose dans le plan de la source : une cartographie de la dose en surface et en profondeur au niveau de la fesse gauche a été obtenue par simulations numériques en associant un code de calcul Monte Carlo et le fantôme voxélisé personnalisé. La dose absorbée à la surface est très élevée (presque 2000 Gy). Le gradient de dose est élevé : les isodoses 20 Gy et 5 Gy sont situées respectivement à $5 \mathrm{~cm}$ et $10 \mathrm{~cm}$ du centre de la lésion. (D) Guide du geste chirurgical par la dosimétrie physique : s'appuyant sur cette cartographie, une exérèse de $5 \mathrm{~cm}$ de profondeur et de $10 \mathrm{~cm}$ de diamètre a été pratiquée au niveau de la fesse gauche.

Dosimetric reconstruction with SESAME: dosimetry guided surgery. (A) Computed Tomography (CT) scans of the patient: 163 slices from mid-abdomen to mid-thigh were used to generate a personalized numerical voxel phantom representative of the patient. (B) View of the voxel phantom of the victim with the source using SESAME software. A voxel phantom with external contours and bone structures was generated and the ${ }^{192}$ Ir source was positioned at $2 \mathrm{~mm}$ from the centre of the skin lesion surface on the left buttock. (C) Dose distribution pattern obtained at the same level as the source plane: a map of the dose distribution on the skin surface and underlying tissues of the buttock was obtained with numerical simulations associating a Monte Carlo code and the personalized voxel phantom. The dose absorbed at the skin surface is very high (almost $2000 \mathrm{~Gy}$ ). The gradient of the dose was very sharp: the $20 \mathrm{~Gy}$ and $5 \mathrm{~Gy}$ isodoses were respectively situated at $5 \mathrm{~cm}$ and $10 \mathrm{~cm}$ from the centre of the lesion. (D) Dosimetry-guided surgery: based on this mapping, a surgical excision measuring $10 \mathrm{~cm}$ diameter in surface and $5 \mathrm{~cm}$ in depth was performed on the left buttock. 


\section{Conclusion}

Suite à un accident radiologique dû à une source d ${ }^{192} \mathrm{Ir}$ provenant d'un gammagraphe, la reconstitution dosimétrique par simulations numériques a été réalisée en tenant compte du scénario décrit par la victime.

Afin, notamment, de définir les contours de l'exérèse envisagée au niveau de la fesse, une cartographie dosimétrique en surface et en profondeur a été établie. Les calculs réalisés avec la géométrie simplifiée ainsi qu'avec le fantôme voxélisé, pour une source placée à $2 \mathrm{~mm}$ de la surface de la peau et un temps d'exposition de 10 minutes, montrent que l'isodose 20 Gy se trouve à environ $5 \mathrm{~cm}$ de profondeur. Une incertitude de plus ou moins 5 minutes sur le temps d'exposition entraîne une variation sur la position de l'isodose $20 \mathrm{~Gy}$ de plus ou moins 1,2 centimètres environ et une variation de plus ou moins $10 \mathrm{~Gy}$ sur la valeur de la dose à $5 \mathrm{~cm}$ de profondeur. S'appuyant sur cette cartographie, une exérèse de $5 \mathrm{~cm}$ de profondeur et de $10 \mathrm{~cm}$ de diamètre a été pratiquée au niveau de la fesse le 5 janvier 2006 par l'équipe de l'hôpital d'instruction des Armées Percy à Clamart (Fig. 10). Cette chirurgie anticipatoire guidée par une reconstitution dosimétrique par simulations numériques constitue une première mondiale pour le traitement des lésions à la suite d'une irradiation localisée.

\section{RÉFÉRENCES}

Cristy M., Eckerman K.F. (1987) Specific absorbed fractions of energy at various ages from internal photon sources, I. Methods, Report Health and Safety Research Division of Oak Ridge National Laboratory, TM-8381/V1.

Furstoss C., Ménard S. (2005) Rapport IRSN DRPH/SDE n 2005-13, Description de la géométrie du fantôme mathématique anthropomorphe utilisé pour la modélisation avec les codes Monte Carlo MCNP et MCNPX.

Lemosquet A., Clairand I., de Carlan L., Franck D., Aubineau-Lanièce I., Bottollier-Depois J.-F. (2004) A computational tool based on voxel geometry for dose reconstruction of a radiological accident due to external exposure, Radiat. Prot. Dosim. 110, 449-454.

Van Riper K. (1997) Sabrina User's Guide, White Rock Science.

Waters L.S. (2002) MCNPX User's Manual, Version 2.4.0, LA-CP-02-408, Los Alamos National Laboratory. 